\title{
Uso de las redes sociales virtuales por los estudiantes de la UAT
}

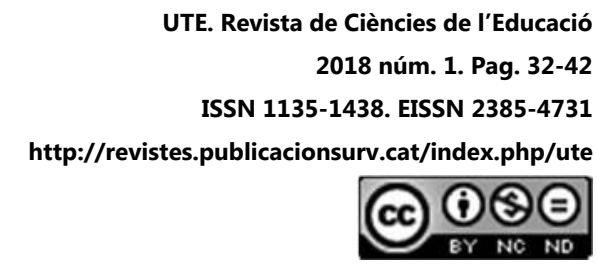

DOI: https://doi.org/10.17345/ute.2018.1

\section{Fernando Leal Rìos, María García Leal, Pedro Carlos Estrada Bellman, Julio Cabero Almenara}

Rebut: 30/05/2018 Acceptat: 20/06/2018

\begin{abstract}
Resumen
Las redes sociales virtuales son las herramientas más representativas del cambio sustancial en la humanidad, además implican una oportunidad importante para generar puentes de comunicación más amigables entre estudiantes, profesores y otros actores relacionados con el ámbito educativo. El objetivo del trabajo fue conocer los hábitos de comunicación en las redes sociales virtuales por estudiantes de la Universidad Autónoma de Tamaulipas (México). La metodología utilizada fue socio-empírica, de tipo transversal y cuantitativa, la muestra estuvo conformada por 250 alumnos de nivel licenciatura de diferentes programas educativos que se imparten en la Universidad Autónoma de Tamaulipas. Los resultados encontrados indican que fue un $33 \%$ de mujeres las que cumplimentaron el cuestionario, el rango de edad de los participantes oscila entre los 18-24 años, los encuestados mencionan que el $40 \%$ de los contenidos que comparten en redes sociales son tareas. Así mismo el $26 \%$ y el $40 \%$ de los alumnos indican que el horario de la mañana y por la noche es en el que usan con más frecuencia las redes sociales, respectivamente. A manera de conclusión se encontró que la red social Facebook, así como el servicio de mensajería instantánea WhatsApp son utilizadas con mayor frecuencia por los estudiantes, así como el uso del celular es el dispositivo más utilizado por los estudiantes en el momento de conectarse.
\end{abstract}

Palabras clave: Uso, Redes sociales virtuales, Estudiantes.

\begin{abstract}
Virtual social networks are the most representative tools of substantial change in humanity. They also imply an important opportunity to create more friendly communication bridges between students, teachers and other actors related to education. The objective of the work was to know the communication habits in the virtual social networks by students of the Autonomous University of Tamaulipas. The methodology used was socio-empirical, of transversal and quantitative type, the sample was conformed by 250 undergraduate students of different educational programs that are impart in the Autonomous University of Tamaulipas. The results found indicate that $33 \%$ of women completed the questionnaire, the age range of the participant's ranges from 18-24 years, respondents mentioned that $40 \%$ of the contents they share in social networks are tasks. Likewise, $26 \%$ and $40 \%$ of students indicate that the morning and evening hours are the ones used by social networks, respectively. By way of conclusion, we found that the social network Facebook as well as the instant messaging service WhatsApp are used more frequently by the students as well as the use of the cell phone is the most used device by the students at the time of connecting.
\end{abstract}

Keywords: Use, Virtual social networks, Students. 


\section{Introducció i objectius del treball}

No estaría mal asumir desde el principio que en los nuevos tiempos que corren, son necesarias nuevas formas de resolverse, nuevas maneras de abordar los problemas y nuevas estructuras para comprenderlos, nuevas estrategias de plantear las relaciones con las personas, y también, y es lo que aquí nos importa, nuevas herramientas de comunicación, que van a requerir que las personas las dominemos, tanto desde un punto de vista instrumental, como sintáctico y semántico para la construcción con ellas de mensajes.

\subsection{Problema/cuestión}

Las redes sociales pueden perfectamente utilizarse en los procesos de formación para construir con ellas entornos donde los estudiantes pueden compartir diferentes objetos de aprendizaje, y pueden de hacerlo en entornos abiertos y flexibles, que le permite al profesor no sólo controlar las actividades y acciones que realizan sus estudiantes, sino también qué personas son las que conforman el entorno de formación, y qué tipo de actividades realizan.

En nuestro caso de estudiantes universitarios, nos interesa conocer, si las actitudes de usos y patrones de interacción dependen del género de la persona, su edad, carrera que se encuentren cursando o frecuencia de utilización.

\subsection{Revisión de la literatura}

La educación aparece como uno de los campos privilegiados de explotación de las posibilidades comunicativas de la red y ante semejantes perspectivas los educadores no podemos quedar atrás indiferentes. Dadas sus posibilidades, el fenómeno recaba la atención desde la investigación ya que la comunidad educativa con sus modos, medios y técnicas necesita adaptarse a una sociedad cada vez más apoyada en las tecnologías de la información y desde esta perspectiva el fenómeno de Internet debe ser analizado, investigado y experimentado para la enseñanza. Ello requiere que tanto profesores como alumnos, deban tener una serie de competencias para saber desenvolverse con las tecnologías que están cambiando nuestros escenarios formativos.

Tal es el impacto que están teniendo las TICs en la sociedad del conocimiento (Cabero, 2008), que ya se habla de alfabetizaciones múltiples, para hacer referencia con ella, a las capacidades que tanto el profesor como el alumno deben tener para desenvolverse en estos nuevos contextos, y donde ya no es suficiente con el dominio lecto-escritores de los códigos tradicionales, sino que se extienden a los lenguajes que emanan de los medios audiovisuales, informáticos y telemáticos.

Según Sánchez y Fernández (2010), gracias a un estudio realizado por la Universidad Camilo José Cela sobre los hábitos de los adolescentes en el uso de las redes sociales, la principal razón para usar este tipo de herramienta es para mantener el contacto con amigos y más lejos aparece otra razón social: "saber de gente con la que no se tiene relación habitual".

Los internautas emplean la web con objetivos diversos que no pueden catalogarse estrictamente como públicos o privados. Entre estos usos destaca la formación de grupos que funcionan como redes para compartir o brindar información de todo tipo, como prestar ayuda en caso de emergencia, ofrecer asistencia legal, socializar los contactos que cada quien establece con diversas instituciones y discutir acerca de asuntos de interés público, colectivo o que están en la agenda de los medios (Winocur, 2006). Así, las redes sociales virtuales son sistemas de relaciones que las personas entablan con amigos/conocidos, llamados contactos, con los cuales se interrelacionan por medio de alguna plataforma electrónica asociada a la Internet (Cortés, 2015), como, por ejemplo, Facebook, Twitter e Instagram, por mencionar algunas. 
Entre los sitios de red social que podemos crear en las redes sociales verticales, es evidente que una de las grandes posibilidades es la de crear una red social que sea utilizada para favorecer o enriquecer los procesos de enseñanza y aprendizaje. Pero además, ya han hecho su aparición, algunos sitios de red social horizontal cuya finalidad es específicamente educativa.

Según Cachia (2008) las redes sociales educativas presentan una serie de ventajas: crean una dimensión nueva de socialización; proporcionan la base para pensar en una formidable herramienta para la educación inclusiva; se están usando como un espacio de encuentro entre los distintos actores del proceso de aprendizaje/enseñanza; sirven de enlace con las empresas que ofrecen empleo; facilitan tareas de inmersión en un entorno lingüístico extranjero por medio de redes o comunidades; dinamizadores y constructoras de una identidad digital.

Así, dependiendo también del uso que hagamos de las redes sociales para la educación, podríamos distinguir entre redes sociales para el desarrollo profesional docente y para la actividad de o para el alumnado.

Teniendo en cuenta que las redes sociales son generalmente utilizadas para la comunicación e interacción entre miembros con intereses comunes no resultaría ningún descubrimiento afirmar que éstas pueden contribuir al desarrollo profesional docente a través del intercambio de ideas y trabajo colaborativo, tal y como afirman Pérez y otros (2010), siendo ésta la primer distinción que realizamos dentro de las redes sociales para la educación.

Hay un punto, sin embargo, que no puede dejar de considerarse: su influencia entre los estudiantes universitarios. Valerio y Valenzuela (2011) han realizado aportaciones destacadas al respecto, con especial atención en dichos sujetos. Algunos estudios recientes, enfocados en los usos y afectaciones de las redes sociales virtuales en entornos escolares de Iberoamérica (Gómez, Roses y Farías, 2012) señalan un reconocimiento del potencial didáctico de la red por parte de los propios estudiantes que, incluso, están dispuestos a participar en este modo de comunicación para difundir y compartir materiales, opiniones y conocimientos (Alonso y Alonso, 2014; Cancelo, 2013; Cortés, 2015).

Las redes sociales virtuales implican una oportunidad importante para generar puentes de comunicación más amigables entre estudiantes, profesores y otros actores relacionados con el ámbito educativo.

Vamos a considerar que nuestros alumnos/as tiene una doble vida, incluso muchas más, pero aquí solo nos interesa aquella que se centre en su vida dentro y fuera del aula, marcada en muchos casos por la presencia o no delas tecnologías y de una cultura mediática, hecho que puede influir y modificar su entorno de aprendizaje. Ahora bien, en estas dos vidas podemos afirmar que se producen algunas contradicciones. En su vida dentro del aula, el alumno/a sigue desarrollando su hemisferio izquierdo, aunque afuera se desarrolla más en un entorno visual. Como dicen las profesoras Aguiar y Farray (2007) hemos pasado de la generación Guttemberg a la generación Videns y por último a la Blade Runner.

\subsection{Propósito}

Conocer las percepciones y los usos que los alumnos universitarios realizan de las redes sociales y determinar estudiar si estas percepciones, vienen influenciado por el género de los estudiantes, edad, frecuencia de uso de las redes sociales, tipo de carrera que cursan.

La hipótesis planteada fue que

No existen diferencias significativas entre el género, edad y tipo de carrera que cursan los alumnos de la UAT, respecto a la frecuencia de uso de las redes sociales. 


\section{Metodología}

El estudio que se realizó sigue la misma estructura y diseño metodológico que el denominado "Capacitación digital de los alumnos de la Unidad Académica Multidisciplinaria de Agronomía y Ciencias (Ciudad Victoria) de la Universidad Autónoma de Tamaulipas (Leal y otros, 2009) y que pretendía analizar las competencias digitales de los estudiantes de la Universidad.

El trabajo de investigación realizado con base al propósito planteado fue un estudio descriptivo, considerado como el punto de partida de otros estudios y estuvo dirigido a determinar "cómo es" o "cómo está" la situación de las variables que se estudian, en este caso, los hábitos de comunicación en las redes sociales virtuales por estudiantes.

Así mismo el estudio se encuentra enmarcado dentro de la sociología empírica, dado que el objetivo de esta estrategia lo constituyen fenómenos sociales "hechos o fenómenos que se presentan de manera más o menos generales en un contexto social determinado" (Canales, 1986; p. 145). Y el diseño del estudio es de carácter transversal y cuantitativo, teniendo como objetivo la descripción tan completa como sea necesario, en un solo tiempo, por única vez y no incluye estudio de los cambios.

La muestra estuvo conformada por 250 alumnos de nivel licenciatura de la Universidad Autónoma de Tamaulipas. El instrumento que se utilizó para la recogida de la información fue un cuestionario de carácter exploratorio que estaba conformado por 35 ítems, donde los primeros 11 ítems, permitían conocer los hábitos de uso y consumo de las redes sociales virtuales por parte de los estudiantes. Para ello, en el cuestionario se preguntó a quienes participaron, entre otras interrogantes, cuáles eran sus redes favoritas, desde qué dispositivos se conectaban y en qué sitios lo hacían.

Para la obtención del índice de fiabilidad, se aplicó el alfa de Cronbach, alcanzando un valor de 0.986, que de acuerdo con Bisquerra (2004), se podría decir que es un índice de fiabilidad "muy alto". Para determinar el tamaño de la muestra, se empleó el programa MACStats versión 2.5, y se consideraron un valor de Alfa de 0.5 y un valor de $p$ y $q$ iguales a 0.5 , lo que dio como resultado un tamaño de la muestra equivalente a 250, el cuestionario se administró al finalizar el curso académico enero-mayo del 2017.

El análisis de la información se realizó por medio de la minería de datos, como técnica para descubrir los patrones de uso de las redes sociales virtuales y su relación con las decisiones de los usuarios y enfocar su atención especialmente en las posibilidades de usar estas redes como herramientas educativas.

Para el análisis, se utilizó el software WEKA (Waikato Environment for Knowledge Analysis) (Witten y Frank, 2005), que es una herramienta visual de libre distribución, desarrollada por los investigadores de la Universidad de Waikato en Nueva Zelanda. WEKA está implementado en el lenguaje de programación Java y opera en diferentes entornos de sistemas operativos.

\section{Resultados}

Los alumnos que cumplimentaron el cuestionario fueron 250 , de los cuales la gran mayoría $(f=216$, 86.5\%) eran hombres y sólo el $13.5 \%(f=34)$ mujeres.

Como podemos observar en la Tabla 1, entre la banda de edad de los 18 años a los 21, nos encontramos con el $80 \%$ de la distribución de los alumnos. Al mismo tiempo, señalar que pocos son los alumnos que tiene más de 30 años ( $f=23,2.4 \%$ ). 


\begin{tabular}{|c|c|c|}
\hline Edad & Frecuencia & Porcentaje \\
\hline 17 & 7 & $2.7 \%$ \\
\hline 18 & 35 & $14.3 \%$ \\
\hline 19 & 32 & $12.8 \%$ \\
\hline 20 & 47 & $18.6 \%$ \\
\hline 21 & 51 & $20.5 \%$ \\
\hline 22 & 40 & $15.9 \%$ \\
\hline 23 & 10 & $3.9 \%$ \\
\hline 24 & 6 & $2.3 \%$ \\
\hline 25 & 4 & $1.6 \%$ \\
\hline 26 & 5 & $2.3 \%$ \\
\hline 27 & 3 & $1.2 \%$ \\
\hline 28 & 3 & $1.2 \%$ \\
\hline 31 & 2 & $0.8 \%$ \\
\hline 35 & 1 & $0.4 \%$ \\
\hline 40 & 3 & $1.2 \%$ \\
\hline 44 & 1 & $0.4 \%$ \\
\hline Total & 250 & $100.0 \%$ \\
\hline & Tabla 1. Edad de los alumnos. \\
\hline
\end{tabular}

Para llevar a cabo el proceso de minería de datos, se conformó una base de datos con 11 atributos, entre los que se consideró información general, como el género del participante, frecuencia con que se conecta a Internet desde lugares como la escuela o la casa, Asimismo, se incluyó la frecuencia de conexión desde diferentes dispositivos, como teléfonos inteligentes, tabletas y computadoras portátiles o de escritorio. Las redes seleccionadas para el proceso de minería de datos fueron las más populares: Facebook, Twitter, YouTube, Instagram, Google + y el servicio de mensajería instantánea WhatsApp, que fue señalado en repetidas ocasione por los informantes, de modo que se incluyó entre los atributos seleccionados.

Previamente, se comentó que el sitio web Alexa posicionaba a Facebook como la red más popular del mundo. Los datos recabados localmente coinciden con dicha aseveración, pero emergió un dato inesperado: la popularidad de WhatsApp, que no constituye propiamente una red, sino un servicio digital de mensajería instantánea que permite crear grupos de contactos -entiéndanse redesespecíficos. Por esta razón, no se incluyó en el diseño original del cuestionario, pero en el estudio piloto los estudiantes voluntarios comentaron que debía incluirse, pues no sólo la utilizaban, sino que la concebían como una red social virtual. Ante esto, los resultados obtenidos fueron que el $27 \%$ de la muestra ubicó WhatsApp como la red con mayor índice de conexión, seguida por Facebook, con el 25 $\%$; YouTube, con el $16 \%$; y Twitter, con el $11 \%$.

La generación de grupos es una de las técnicas más recurridas en la minería de datos. En este trabajo se experimentó con el algoritmo SimpleKMeans (MacQueen, 1967), el cual ha sido utilizado con éxito en diversas investigaciones.

Para un análisis más preciso se conformaron dos grupos de estudiantes de todos los que cumplimentaron el cuestionario, en los que destacan, como atributos mutuamente excluyentes, el 
género, la frecuencia de conexión en la escuela, la frecuencia de conexión en casa y la red social virtual YouTube.

Los valores reportados en el Tabla 2, señalan claras diferencias de género y en la población que participó en el experimento. En general, tanto hombres (216) como mujeres (34) manifiestan poca conexión a la Internet desde la escuela. Este comportamiento se debe, en parte, a las restricciones de conexión a la red inalámbrica en dispositivos móviles dentro de los campus de la UAT.

\begin{tabular}{|l|c|c|}
\hline \multicolumn{1}{|c|}{ Atributo } & Grupo 1 & Grupo 2 \\
& 34 & 216 \\
\hline Género & Femenino & Masculino \\
\hline Frecuencia de conexión en la escuela & Nunca & A veces \\
\hline $\begin{array}{l}\text { Frecuencia de conexión en casa desde computadora } \\
\text { portátil o de escritorio }\end{array}$ & Siempre & Frecuentemente \\
\hline Red social virtual YouTube & No & Sí \\
\hline
\end{tabular}

Tabla 2. Generación de dos grupos de estudiantes con el algoritmo SimpleKmeans por medio del programa WEKA

En cambio, la frecuencia de conexión en casa por medio de una computadora portátil o de escritorio es permanente entre las mujeres y sólo frecuente entre los hombres. Por último, destaca la presencia de la plataforma de videos YouTube, donde se observa que los hombres la consideran como una de sus favoritas.

Posteriormente, se generó con la información recabada tres grupos de estudiantes en los que destacan como atributos mutuamente excluyentes la frecuencia de conexión en la escuela y la frecuencia de conexión en casa mediante una computadora portátil o de escritorio (Tabla 3).

\begin{tabular}{|l|c|c|c|}
\hline \multicolumn{1}{|c|}{ Atributo } & $\begin{array}{c}\text { Grupo 1 } \\
18\end{array}$ & $\begin{array}{c}\text { Grupo 2 } \\
17\end{array}$ & $\begin{array}{c}\text { Grupo 3 } \\
10\end{array}$ \\
\hline Frecuencia de conexión en la escuela & Nunca & A veces & Rara vez \\
\hline $\begin{array}{l}\text { Frecuencia de conexión en casa desde } \\
\text { computadora portátil o de escritorio }\end{array}$ & Siempre & Frecuentemente & Nunca \\
\hline
\end{tabular}

Tabla 3. Generación de tres grupos de estudiantes con el algoritmo SimpleKmeans por medio del programa WEKA

Los valores reportados en la Tabla 3, identifican que, en general, los alumnos se conectan poco en la escuela. Hay incluso un grupo de alumnos que manifestó que nunca se conecta allí. En cuanto a la frecuencia de conexión en casa por medio de una computadora portátil o de escritorio, la mayoría de los alumnos que participó en el experimento lo hacen siempre o frecuentemente.

La generación de reglas de asociación es otra de las técnicas de la minería de datos. En este trabajo se implementaron dos algoritmos para estudiar los comportamientos de los informantes con respecto a sus preferencias por una u otra red.

El primer algoritmo utilizado fue el Predictive Apriori, del cual se recuperaron 20 reglas cuyas alfas de confiabilidad eran de 0.95 . En el presente estudio se describen tres, las cuales se eligieron por su nivel de confianza y profundidad. Entre los resultados, destaca la gran popularidad del servicio de mensajería 
instantánea WhatsApp, en relación con los diferentes lugares y dispositivos de conexión, así como de las redes señaladas como predilectas (Tabla 4).

\begin{tabular}{|c|c|c|}
\hline Regla generada por medio de WEKA & Interpretación en lenguaje natural & $\begin{array}{l}\text { Índice de } \\
\text { confianza }\end{array}$ \\
\hline $\begin{array}{l}\text { Dispositivo Smartphone = Siempre } \\
\text { Favorita Facebook = Si } \\
\text { Favorita Twitter = SI } \\
\text { Favorita Google+ = No } 67 \\
\text { Favorita WhatsApp = Si } 67\end{array}$ & $\begin{array}{l}\text { Si el usuario siempre accede a la } \\
\text { Internet desde un teléfono inteligente } \\
\text { y Facebook y Twitter son sus redes } \\
\text { favoritas, pero no Google+, entonces } \\
\text { tiene preferencia por WhatsApp }\end{array}$ & (0.99) \\
\hline $\begin{array}{l}\text { Género = Femenino } \\
\text { Dispositivo Smartphone= } \\
\text { Frecuentemente } \\
\text { Favorita Facebook = Si } \\
\text { Favorita Instagram = SI } 20 \\
\text { Favorita WhatsApp = Si } 20\end{array}$ & $\begin{array}{l}\text { Si pertenecen al género femenino, se } \\
\text { conectan frecuentemente a la Internet } \\
\text { desde un teléfono inteligente y entre } \\
\text { sus redes favoritas Facebook e } \\
\text { Instagram entonces también tiene } \\
\text { predilección por WhatsApp }\end{array}$ & $(0.99)$ \\
\hline $\begin{array}{l}\text { Dispositivo Tablet }=\text { Siempre } \\
\text { Favorita Facebook }=\text { Si } \\
\text { Favorita Instagram }=\text { SI } 49 \\
\text { Favorita WhatsApp }=\text { Si } 49\end{array}$ & $\begin{array}{l}\text { Si el usuario siempre accede siempre a } \\
\text { la Internet desde una tableta y entre } \\
\text { sus redes favoritas están Facebook, } \\
\text { Twitter e Instagram son sus redes } \\
\text { favoritas, pero no Google+, entonces } \\
\text { WhatsApp también esta entres sus } \\
\text { favoritas }\end{array}$ & $(0.99)$ \\
\hline
\end{tabular}

Tabla 4. Ejemplo de la descripción de tres reglas generadas mediante la aplicación de algoritmo Predictive Apriori

En los resultados de la Tabla 4, se observa la destacada presencia de WhatsApp entre las predilecciones de los usuarios, aspecto que se desarrollará más adelante.

El segundo algoritmo empleado fue el Apriori, que arrojó 20 reglas de asociación, cuyas alfas también alcanzaron el 0.95 de confiabilidad. A diferencia de los resultados expuestos por el Predictive Apriori, en estas reglas se observa más presencia de la red social Facebook para el esbozo de patrones en la Tabla 5. De la misma manera que en el caso anterior, a continuación, se explican algunas de las reglas más destacadas que resultaron del algoritmo Apriori.

En la Tabla 5, se observa que se refrenda el gran impacto de WhatsApp entre la población estudiada, incluso por encima de la red social virtual más popular del mundo, que es Facebook. Este resultado incluye un par de ideas que deben destacarse: la indiferenciación de los estudiantes con respecto de 
una red social virtual y un servicio de mensajería instantánea. También se aprecia el gran poder de penetración de tecnologías como el teléfono inteligente en la vida cotidiana de los individuos. Si bien WhatsApp cuenta, actualmente, con aplicaciones para computadora, su principal dispositivo continúa siendo el teléfono inteligente.

\begin{tabular}{|c|c|c|}
\hline Regla generada por medio de WEKA & Interpretación en lenguaje natural & $\begin{array}{l}\text { Índice de } \\
\text { confianza }\end{array}$ \\
\hline $\begin{array}{l}\text { Conexión casa = Siempre } \\
\text { Favorita Facebook }=\mathrm{Si} \\
\text { Favorita YouTube }=\text { Si } 19 \\
\text { Favorita WhatsApp }=18\end{array}$ & $\begin{array}{l}\text { Si el usuario accede a la Internet } \\
\text { siempre desde su casa y prefiere } \\
\text { WhatsApp y YouTube, entonces } \\
\text { Facebook también es una de sus redes } \\
\text { favoritas }\end{array}$ & $(0.92)$ \\
\hline $\begin{array}{l}\text { Favorita YouTube }=\mathrm{Si} \\
\text { Favorita WhatsApp }=\mathrm{Si} \\
\text { Favorita Instagram }=\text { No } 17 \\
\text { Favorita Facebook }=63\end{array}$ & $\begin{array}{l}\text { Si YouTube y WhatsApp se encuentran } \\
\text { entre las predilectas del usuario, pero } \\
\text { no Instagram, entonces Facebook } \\
\text { destaca entre sus redes favoritas } \\
\text { también }\end{array}$ & $(0.92)$ \\
\hline $\begin{array}{l}\text { Género = Femenino } \\
\text { Favorita Facebook }=20 \\
\text { Favorita WhatsApp }=19\end{array}$ & $\begin{array}{l}\text { Si pertenecen al género femenino y su } \\
\text { red predilecta es Facebook, entonces } \\
\text { también tienen predilección por } \\
\text { WhatsApp }\end{array}$ & $(0.90)$ \\
\hline
\end{tabular}

Tabla 5. Ejemplo de la descripción de tres reglas generadas mediante la aplicación del algoritmo Apriori.

\section{Discusión y conclusiones}

Del estudio que hemos realizado, se pueden extraer una serie de discusiones, que se presentar a continuación, pero antes es válido señalar que, respecto al grado de alcance de los objetivos en el trabajo, todos han sido alcanzados y cumplidos.

Del mismo modo se presenta la evaluación e interpretación de los datos obtenidos en la sección anterior, refiriéndolos especialmente a los objetivos y otras investigaciones realizadas y las conclusiones del trabajo.

Con respecto a la presencia de la tecnología informática y de Internet en los domicilios de los alumnos, nos hemos encontrado, que en el primer caso el 74.0 \% tienen acceso al mismo, y en el segundo el 53.1 $\%$. Porcentajes, que, si bien no son bajos, nos deben hacer reflexionar a que la Universidad debe adoptar medidas, para facilitar su utilización por parte de los estudiantes, y que no vaya a convertirse la realización de acciones formativas apoyadas en estas tecnologías en elemento de discriminación para los estudiantes más desfavorecidos.

Por otra parte, señalar que, en el estudio expone con completa claridad que, el disponer en los domicilios de las tecnologías -en este caso de la computadora, conexión a Internet y laptop- ha repercutido para 
que los alumnos se encuentren más competentes y capacitados con las mismas. Hecho que también se encontró en otros trabajos (Bullón, 2008; Cabero, Llorente y Puentes, 2008; Llorente, 2008).

Entre los resultados principales relacionados con los hábitos de comunicación por medio de las redes sociales, se observan claras tendencias en los hábitos de conexión, la preferencia por alguna red social virtual y los dispositivos principales. En la aplicación del algoritmo SimpleKMeans destaca la generación de grupos con hábitos de conexión a la Internet desde una computadora portátil o de escritorio en los rangos siempre y frecuentemente. Asimismo, se aprecia que YouTube figura como la red social virtual favorita de un grupo en particular.

Con el mismo algoritmo se encontraron tres grupos de estudiantes: uno que nunca se conecta a la Internet desde la escuela; otro que lo hace a veces $y$, finalmente, otro más que lo hace rara vez. Lo anterior se traduce en que la escuela es uno de los sitios menos recurrentes para conectarse a la Internet, lo cual se contrapone con los resultados de la conexión desde el hogar. En este último caso, se observan tres grupos: uno que se conecta siempre, otro que lo hace frecuentemente y un tercero que nunca lo hace. Esto implica una condición socioeconómica importante, pues podría significar la existencia de un grupo de individuos cuyo poder adquisitivo es suficiente para contratar un servicio de Internet domiciliario y de otro que no puede hacerlo.

Con respecto a los resultados de los algoritmos de asociación (Predictive Apriori y Apriori), se aprecia que las redes más populares son Facebook, YouTube y Twitter, pero los estudiantes reconocen el servicio de mensajería instantánea WhatsApp como una red social virtual, y ocupa un lugar importante entre las preferencias de los usuarios. Esto revela una idea importante: si una tecnología particular es útil y amigable para la comunicación cotidiana de los individuos, la utilizarán, independientemente de si es una red o un servicio de mensajería instantánea, como ocurre, en este caso, con WhatsApp.

En términos generales, con respecto a los hallazgos encontrados mediante la aplicación de la minería de datos, las reglas señaladas sugieren condiciones importantes. En cuanto a los dispositivos de conexión, se aprecia que, pese a la gran aceptación del teléfono inteligente en la vida cotidiana, la presencia de la computadora portátil o de escritorio como dispositivo de conexión a la Internet sigue siendo la predilección de los universitarios.

Por otro lado, la alta popularidad de WhatsApp también sugiere otro hallazgo relacionado directamente con el teléfono inteligente. Para contar con la aplicación WhatsApp es necesario que el usuario tenga un equipo con un mínimo de características tecnológicas, en este caso, la conexión a la Internet. WhatsApp es un servicio de uso gratuito, pero su costo en consumo de megas, como parte de un plan telefónico, es mínimo. Esta característica constituye uno de sus principales atractivos y desplaza al tradicional servicio de mensajería que distinguía a los teléfonos celulares, pues, actualmente, cada mensaje tiene un costo superior a los $\$ 2.00$ pesos $\mathrm{MN}$, una suma incosteable para muchos públicos, sobre todo el de los estudiantes.

Esta situación también se repite con Facebook, pues al contar con una aplicación para dispositivos móviles, como las tabletas y teléfonos inteligentes, permite el envío y la recepción de mensajes de manera gratuita, como ocurre con WhatsApp. Se infiere que una de las razones por las cuales los estudiantes pudieron homologar una y otra en la categoría de red social virtual se debió a que WhatsApp permite crear listas de difusión o grupos específicos, con los cuales pueden compartir información con un colectivo de usuarios específicos, como también lo permite Facebook, y esto podría entenderse como una forma de red. En todo caso, lo importante es el uso que las personas le dan a estas redes, así como la popularidad que han adquirido. 


\section{Referencias bibliográficas}

Aguiar, V.M. y Farray, J.I. (2007). Nuevas Tecnologías Aplicadas a la Educación. Las Palmas de G.C.: Servicio de publicaciones de la ULPGC

Alonso, S., y Alonso, M. (2014). Las redes sociales en las universidades españolas. Revistas de Comunicación Vivat Academia, volumen (126), 54-62

Bisquerra, R. (coord.) (2004). Metodología de la investigación educativa. Madrid. La Muralla Bullon, A. (2008). Alfabetización digital de los estudiantes de la Facultad de Odontología de la Universidad de Sevilla. Sevilla: Grupo de Investigación Didáctica.

Cabero, J. (2008). La alfabetización digital de los alumnos. Competencias digitales para el siglo XXI. Revista de Pedagogía, volumen (2), 7-28.

Cabero, J., Llorente, M. C. y Puentes, A. (2008). Alfabetización Digital: Un estudio en la Pontificia Universidad Católica Madre y Maestra. Sevilla: Fortic.

Cachia, R. (2008). Los sitios de creación de redes. Aspectos sociales. Revista Telos, 76. http://sociedadinformacion.fundacion.telefonica.com/telos/articulocuaderno.asp@idarticulo\%3D4\&rev \%3D76.htm (Consultado el 3 de julio de 2018).

Canales, F.H. (1986). Metodología de la Investigación. Manual para el Desarrollo de personal de Salud. México. Limusa.

Cancelo, M. (2013). Estrategias comunicativas en redes sociales. Estudio comparativo entre las universidades de España y México. Historia y Comunicación Social, volumen (18), 423-435.

Cortes, R. (2015). Interacción en Redes Sociales Virtuales entre estudiantes de Licenciatura. Una aproximación con fines pedagógicos. Revista Iberoamericana de Producción Académica y Gestión Educativa, 2015, volumen (1), Recuperado de: http://www.pag.org.mx/index.php/PAG/article/view/107/155

Gómez. M, Roses, S., y Farías, P. (2012). El uso académico de las redes sociales en universitarios. Comunicar, volumen 19(38), 131-138.

Leal, R.F. Cebero, A. J. Lucero, M. F.A. y Llorente, M.C. (2009). Capacitación digital de los alumnos de la Unidad Académica Multidisciplinaria de Agronomía y Ciencias (Ciudad Victoria) de la Universidad Autónoma de Tamaulipas. Sevilla: Fortic.

Llorente, M.C. (2008). La formación semipresencial a través de redes telemáticas (blended learning). Barcelona: DaVinci.

MacQueen, J. (1967). Some methods for classification and analysis of multivariate observations. En: Proceedings of the fifth Berkeley symposium on mathematical statistics and probability, volumen (1), Statistics (pp. 281-297). Berkeley, CA: University of California, Bekeley

Pérez, T., Castillo E. y Marquina R. (2010). Las redes sociales y el desarrollo profesional del docente. Revista cognición, http://www.cognicion.net/index.php?option=com_content\&task=view\&id=322\&/temid=228 (Consultado el 3 de julio de 2018). 
Sánchez, A. y Fernández, M.P. (2010): Informe Generación 2.0. Hábitos de los adolescentes en el uso de las redes sociales. http://estaticos.elmundo.es/2010/07/06Generacion20.pdf (Consultado el 3 de julio de 2018)

Valerio, G y Valenzuela, R. (2011). Redes sociales y estudiantes universitarios. Del nativo digital al informívoro saludable. El profesional de la información, volumen 20(6), 667-670

Witten, I. H. y Frank, E. (2005). Data Mining: Practical machine learning tools and techniques. San Francisco, CA: Morgan Kaufmann

Winocur, R. (2006). Internet en la vida cotidiana de los jóvenes. Revista Mexicana de Sociología, volumen 68(3), 551-580 